\title{
Cohen-Lenstra sums over local rings
}

\author{
par Christian WitTmann
}

RÉSumÉ. On étudie des séries de la forme $\sum_{M}\left|\operatorname{Aut}_{R}(M)\right|^{-1}|M|^{-u}$, où $R$ est un anneau commutatif local et $u$ est un entier non-negatif, la sommation s'étendant sur tous les $R$-modules finis, à isomorphisme prés. Ce problème est motivé par les heuristiques de Cohen et Lenstra sur les groupes des classes des corps de nombres, où de telles sommes apparaissent. Si $R$ a des propriétés additionelles, on reliera les sommes ci-dessus à une limite de fonctions zêta des modules libres $R^{n}$, ces fonctions zêta comptant les sous- $R$-modules d'indice fini dans $R^{n}$. En particulier on montrera que cela est le cas pour l'anneau de groupe $\mathbb{Z}_{p}\left[C_{p^{k}}\right]$ d'un groupe cyclique d'ordre $p^{k}$ sur les entiers $p$-adiques. Par conséquant on pourra prouver une conjecture de [5], affirmant que la somme ci-dessus correspondante à $R=\mathbb{Z}_{p}\left[C_{p^{k}}\right]$ et $u=0$ converge. En outre on considère des sommes raffinées, où $M$ parcourt tous les modules satisfaisant des conditions cohomologiques additionelles.

Abstract. We study series of the form $\sum_{M}\left|\operatorname{Aut}_{R}(M)\right|^{-1}|M|^{-u}$, where $R$ is a commutative local ring, $u$ is a non-negative integer, and the summation extends over all finite $R$-modules $M$, up to isomorphism. This problem is motivated by Cohen-Lenstra heuristics on class groups of number fields, where sums of this kind occur. If $R$ has additional properties, we will relate the above sum to a limit of zeta functions of the free modules $R^{n}$, where these zeta functions count $R$-submodules of finite index in $R^{n}$. In particular we will show that this is the case for the group ring $\mathbb{Z}_{p}\left[C_{p^{k}}\right]$ of a cyclic group of order $p^{k}$ over the $p$-adic integers. Thereby we are able to prove a conjecture from [5], stating that the above sum corresponding to $R=\mathbb{Z}_{p}\left[C_{p^{k}}\right]$ and $u=0$ converges. Moreover we consider refined sums, where $M$ runs through all modules satisfying additional cohomological conditions. 


\section{Introduction}

A starting point for the problem investigated in this article is the following remarkable identity, published by Hall in 1938 [6]. If $p$ is a prime number, then

$$
\sum_{G}|\operatorname{Aut}(G)|^{-1}=\sum_{G}|G|^{-1},
$$

where $G$ runs through all finite abelian $p$-groups, up to isomorphism. Here we will consider a more general problem. Put

$$
\mathcal{S}(R ; u)=\sum_{M}\left|\operatorname{Aut}_{R}(M)\right|^{-1}|M|^{-u},
$$

where $R$ is a commutative ring, $u$ is a non-negative integer, and the sum extends over all finite $R$-modules, up to isomorphism. By $\operatorname{Aut}_{R}(M)$ we denote the group of $R$-automorphisms of $M$. Sums of this kind occur in Cohen-Lenstra heuristics on class groups of number fields (cf. [2], [3]), so we call $\mathcal{S}(R ; u)$ a Cohen-Lenstra sum.

We want to evaluate these series in certain cases. While in [2], [3] $R$ is a maximal order of a finite dimensional semi-simple algebra over $\mathbb{Q}$, we will assume that $R$ is a local ring. We will mainly focus on the case $R=\mathbb{Z}_{p}\left[C_{p^{k}}\right]$, the group ring of a cyclic group of $p$-power order over the $p$-adic integers, which is a non-maximal order in the $\mathbb{Q}_{p}$-algebra $\mathbb{Q}_{p}\left[C_{p^{k}}\right]$.

In particular we are able to prove a conjecture of Greither stated in [5]:

$$
\mathcal{S}\left(\mathbb{Z}_{p}\left[C_{p^{k}}\right] ; 0\right)=\sum_{M}\left|\operatorname{Aut}_{\mathbb{Z}_{p}\left[C_{p^{k}}\right]}(M)\right|^{-1}=\left(\prod_{j=1}^{\infty} \frac{1}{1-p^{-j}}\right)^{k+1} .
$$

This fills a gap concerning the sums $\mathcal{S}\left(\mathbb{Z}_{p}[\Delta] ; 0\right)$ for an arbitrary $p$-group $\Delta$, for Greither showed in $[5]$ that $\mathcal{S}\left(\mathbb{Z}_{p}[\Delta] ; 0\right)$ diverges if $\Delta$ is non-cyclic.

The outline of the paper is as follows. In section 2 we introduce the basic notions concerning Cohen-Lenstra sums over arbitrary local rings, and we will relate these sums to limits of zeta functions. If $V$ is an $R$-module, the zeta function of $V$ is defined as the series

$$
\zeta_{V}(s)=\sum_{U \subseteq V}[V: U]^{-s} \in \mathbb{R} \cup\{\infty\},
$$

where $s \in \mathbb{R}$ and $\zeta_{V}(s)=\infty$ iff the series diverges. The summation extends over all $R$-submodules $U$ of $V$ such that the index $[V: U]$ is finite. The main theorem of that section is 2.6, which states that under certain conditions the Cohen-Lenstra sum $\mathcal{S}(R ; u)$ can be computed if one has enough information on the zeta functions of $R^{n}$, viz

$$
\mathcal{S}(R ; u)=\lim _{n \rightarrow \infty} \zeta_{R^{n}}(n+u) .
$$


In section 3 we derive some results on the zeta function of $V$ at $s=n$, where $V$ is a $\mathbb{Z}_{p}\left[C_{p^{k}}\right]$-module such that $p \mathbb{Z}_{p}\left[C_{p^{k}}\right]^{n} \subseteq V \subseteq \mathbb{Z}_{p}\left[C_{p^{k}}\right]^{n}$. The main ingredient will be a "recursion formula" from [14] for these zeta functions. These results will be applied in section 4 in order to prove Greither's conjecture.

In section 5 we discuss refinements of Cohen-Lenstra sums with respect to the ring $\mathbb{Z}_{p}\left[C_{p}\right]$, where the summation extends only over those modules $M$ having prescribed Tate cohomology groups $\widehat{H}^{i}\left(C_{p}, M\right)$. This has some applications, e.g. in [5], where the case of cohomologically trivial modules is treated, and in [15], where sums of this kind occur as well, when studying the distribution of $p$-class groups of cyclic number fields of degree $p$.

We will use the following notations in the sequel. $\mathbb{N}$ is the set of nonnegative integers, $\mathbb{R}_{+}$the set of non-negative real numbers, $p$ denotes a prime number, $q=p^{-1}$, and $\mathbb{Z}_{p}$ is the ring of $p$-adic integers. We remark that the completion $\mathbb{Z}_{p}$ could be replaced by $\mathbb{Z}_{(p)}$, the localization of $\mathbb{Z}$ at $p$, throughout. If $m \in \mathbb{N} \cup\{\infty\}$, then

$$
(q)_{m}:=\prod_{j=1}^{m}\left(1-q^{j}\right)
$$

note that the product converges for $m=\infty$ because of $0<q<1$. If $l, m \in \mathbb{N}$, we let $\left[\begin{array}{c}m \\ l\end{array}\right]_{p}$ denote the number of $l$-dimensional subspaces of an $m$-dimensional vector space over the finite field $\mathbb{F}_{p}$. It is well-known that

$$
\left[\begin{array}{c}
m \\
l
\end{array}\right]_{p}=\frac{\left(p^{m}-1\right)\left(p^{m}-p\right) \ldots\left(p^{m}-p^{l-1}\right)}{\left(p^{l}-1\right)\left(p^{l}-p\right) \ldots\left(p^{l}-p^{l-1}\right)}=p^{l(m-l)} \frac{(q)_{m}}{(q)_{l}(q)_{m-l}} .
$$

This paper is part of my doctoral thesis. I am indebted to my advisor Prof. Cornelius Greither for many fruitful discussions and various helpful suggestions.

\section{Cohen-Lenstra sums and zeta functions}

Let $R$ be a commutative ring.

Definition 2.1. Let $u \in \mathbb{N}$. The Cohen-Lenstra sum of $R$ with respect to $u$ is defined as

$$
\mathcal{S}(R ; u):=\sum_{M}\left|\operatorname{Aut}_{R}(M)\right|^{-1}|M|^{-u} \in \mathbb{R}_{+} \cup\{\infty\},
$$

where the sum extends over all finite $R$-modules, up to isomorphism. In the sequel, all sums over finite $R$-modules are understood to extend over modules up to isomorphism, without further mention. We denote by $\nu(M)$ 
the minimal number of generators of the finite $R$-module $M$, and we put

$$
\begin{aligned}
\mathcal{S}_{n}(R ; u) & :=\sum_{\substack{M \\
\nu(M)=n}}\left|\operatorname{Aut}_{R}(M)\right|^{-1}|M|^{-u}, \\
\mathcal{S}_{\leq n}(R ; u) & :=\sum_{\substack{M \\
\nu(M) \leq n}}\left|\operatorname{Aut}_{R}(M)\right|^{-1}|M|^{-u} .
\end{aligned}
$$

The following notations will be useful.

Notations. If $A, B$ are $R$-modules, we let

$$
\operatorname{Hom}_{R}^{\text {sur }}(A, B):=\left\{\psi \in \operatorname{Hom}_{R}(A, B) \mid \psi \text { surjective }\right\} .
$$

If $M$ is a finite $R$-module with $\nu(M) \leq n$, there is a positive integer $n$ such that $M$ is of the form $M \cong R^{n} / U$ for some $R$-submodule $U$ of finite index in $R^{n}$. We set

$$
\lambda_{n}^{R}(M):=\left|\left\{U \subseteq R^{n} \mid R^{n} / U \cong M\right\}\right|
$$

and

$$
s_{n}^{R}(M):=\left|\operatorname{Hom}_{R}^{\mathrm{sur}}\left(R^{n}, M\right)\right| .
$$

The following lemma, and also Lemma 2.4, are well-known (cf. [2, Prop. 3.1]). However, we give the simple arguments for the reader's convenience.

Lemma 2.2. $\lambda_{n}^{R}(M)=s_{n}^{R}(M)\left|\operatorname{Aut}_{R}(M)\right|^{-1}$ for any finite $R$-module $M$.

Proof. Each $U \subseteq R^{n}$ satisfying $R^{n} / U \cong M$ has the form $U=\operatorname{ker}(\psi)$ for some surjective $\psi \in \operatorname{Hom}_{R}\left(R^{n}, M\right)$. On the other hand, if $\psi_{1}, \psi_{2} \in$ $\operatorname{Hom}_{R}^{\text {sur }}\left(R^{n}, M\right)$, then

$$
\operatorname{ker}\left(\psi_{1}\right)=\operatorname{ker}\left(\psi_{2}\right) \Longleftrightarrow \psi_{1}=\rho \circ \psi_{2}
$$

for some $\rho \in \operatorname{Aut}_{R}(M)$, and this proves the lemma.

Lemma 2.3. $\mathcal{S}_{\leq n}(R ; u)=\sum_{U \subseteq R^{n}} s_{n}^{R}\left(R^{n} / U\right)^{-1}\left[R^{n}: U\right]^{-u}$, where the sums extends over all $R$-submodules $U$ of finite index in $R^{n}$.

Proof. Let $M$ be a finite $R$-module with $\nu(M) \leq n$. Then $M=R^{n} / U$ for some $U \subseteq R^{n}$, and there are $\lambda_{n}^{R}(M)=\lambda_{n}^{R}\left(R^{n} / U\right)$ possible $U^{\prime}$ with $M \cong R^{n} / U^{\prime}$. Hence the preceding lemma implies

$$
\begin{aligned}
\mathcal{S}_{\leq n}(R ; u) & =\sum_{U \subseteq R^{n}}\left|\operatorname{Aut}_{R}\left(R^{n} / U\right)\right|^{-1} \lambda_{n}^{R}\left(R^{n} / U\right)^{-1}\left|R^{n} / U\right|^{-u} \\
& =\sum_{U \subseteq R^{n}} s_{n}^{R}\left(R^{n} / U\right)^{-1}\left[R^{n}: U\right]^{-u} .
\end{aligned}
$$


Note that the equality in Lemma 2.3 in an equality in $\mathbb{R}_{+} \cup\{\infty\}$ (as are all equalities dealing with Cohen-Lenstra sums in this article).

From now on we assume that $R$ is a local ring with maximal ideal $J$ and residue class field $\mathbb{F}_{p}$. We set

$$
q=p^{-1} .
$$

The restriction to prime fields is not essential. We could just as well suppose that the residue class field of $R$ is an arbitrary finite field $\mathbb{F}_{p^{\alpha}}$. Then all results of this article are still valid if we accordingly set $q=p^{-\alpha}$.

For local rings the calculation of $s_{n}^{R}(M)$ is not difficult. Suppose that $M$ is an $R$-module with $\nu(M) \leq n$. Then

$$
\nu(M)=\operatorname{dim}_{R / J}(M / J M) \in\{0, \ldots, n\}
$$

by Nakayama's Lemma.

Lemma 2.4. $s_{n}^{R}(M)=|M|^{n} \frac{(q)_{n}}{(q)_{n-r}}$, where $r:=\nu(M)$.

Proof. The following equivalence holds for $\psi \in \operatorname{Hom}_{R}\left(R^{n}, M\right)$, by Nakayama's Lemma:

$$
\psi \text { surjective } \Longleftrightarrow \bar{\psi}:(R / J)^{n} \rightarrow M / J M \text { surjective },
$$

where $\bar{\psi}$ is induced by reduction mod $J$. Thus

$$
\begin{aligned}
s_{n}^{R}(M) & =\left|\operatorname{Hom}_{\mathbb{F}_{p}}^{\operatorname{sur}_{p}}\left(\mathbb{F}_{p}^{n}, \mathbb{F}_{p}^{r}\right)\right|\left|\left\{\psi \in \operatorname{Hom}_{R}\left(R^{n}, M\right) \mid \bar{\psi}=0\right\}\right| \\
& =\left(p^{n}-1\right) \ldots\left(p^{n}-p^{r-1}\right)|J M|^{n} \\
& =p^{r n} \frac{(q)_{n}}{(q)_{n-r}}\left(\frac{|M|}{|M / J M|}\right)^{n} \\
& =|M|^{n} \frac{(q)_{n}}{(q)_{n-r}} .
\end{aligned}
$$

Theorem 2.5. $\quad$ a) $\mathcal{S}_{n}(R ; u)=\frac{q^{n(n+u)}}{(q)_{n}} \zeta_{J^{n}}(n+u)$.

b) $\mathcal{S}(R ; u)=\sum_{n=0}^{\infty} \frac{q^{n(n+u)}}{(q)_{n}} \zeta_{J^{n}}(n+u)$.

Proof. It suffices to prove a). If $M \cong R^{n} / U$ for some $U \subseteq R^{n}$, then

$$
\nu(M)=\operatorname{dim}(M / J M)=\operatorname{dim}\left(R^{n} /\left(U+J^{n}\right)\right) .
$$

Therefore $\nu(M)=n$ if and only if $U \subseteq J^{n}$. In an analogous manner as in the proof of Lemma 2.3 we infer

$$
\mathcal{S}_{n}(R ; u)=\sum_{U \subseteq J^{n}} s_{n}^{R}\left(R^{n} / U\right)^{-1}\left[R^{n}: U\right]^{-u},
$$


and using the preceding lemma we get

$$
\mathcal{S}_{n}(R ; u)=\frac{1}{(q)_{n}} \sum_{U \subseteq J^{n}}\left[R^{n}: U\right]^{-(n+u)}=\frac{q^{n(n+u)}}{(q)_{n}} \zeta_{J^{n}}(n+u) .
$$

Examples. a) $R:=\mathbb{F}_{p}$.

Then $J=0$ and

$$
\mathcal{S}\left(\mathbb{F}_{p} ; u\right)=\sum_{n=0}^{\infty} \frac{q^{n(n+u)}}{(q)_{n}} .
$$

In particular, if $u=0$ or $u=1$ the identities of Rogers-Ramanujan (cf. [7, Th. 362, 363]) imply

$$
\begin{aligned}
& \mathcal{S}\left(\mathbb{F}_{p} ; 0\right)=\prod_{m=0}^{\infty} \frac{1}{\left(1-q^{5 m+1}\right)\left(1-q^{5 m+4}\right)} \\
& \mathcal{S}\left(\mathbb{F}_{p} ; 1\right)=\prod_{m=0}^{\infty} \frac{1}{\left(1-q^{5 m+2}\right)\left(1-q^{5 m+3}\right)} .
\end{aligned}
$$

b) Let $R$ be a discrete valuation ring with residue class field $\mathbb{F}_{p}$.

Then $J \cong R$, and it is well-known that

$$
\zeta_{R^{n}}(s)=\prod_{j=0}^{n-1}\left(1-p^{j-s}\right)^{-1}
$$

(cf. $[1, \S 1])$, whence

$$
\mathcal{S}(R ; u)=\sum_{n=0}^{\infty} \frac{q^{n(n+u)}(q)_{u}}{(q)_{n}(q)_{n+u}}=\frac{(q)_{u}}{(q)_{\infty}} .
$$

This result is also proved in [2, Cor. 6.7].

By Theorem 2.5 we are able to compute Cohen-Lenstra sums in some cases, provided we know the zeta functions of $J^{n}$ for $n \in \mathbb{N}$. As we will see in the next section, it may be difficult to calculate $\zeta_{J^{n}}(n+u)$, whereas it is much easier to determine the values $\zeta_{R^{n}}(n+u)$. In these situations the following theorem is useful.

Theorem 2.6. Let $u \in \mathbb{N}$, and recall that $R$ is a local ring. Then:

a) $\mathcal{S}(R ; u)$ converges $\Longleftrightarrow$ The sequence $\left(\zeta_{R^{n}}(n+u)\right)_{n \in \mathbb{N}}$ is bounded.

b) If the sequence $\left(\zeta_{R^{n}}(n+u-1)\right)_{n \in \mathbb{N}}$ is bounded, then

$$
\mathcal{S}(R ; u)=\lim _{n \rightarrow \infty} \zeta_{R^{n}}(n+u) .
$$


Proof. a) The assertion follows from

$$
\begin{aligned}
\zeta_{R^{n}}(n+u) & =\sum_{r=0}^{n} \sum_{\substack{U \subseteq R^{n} \\
\nu\left(R^{n} / U\right)=r}}\left[R^{n}: U\right]^{-(n+u)} \\
& \leq \sum_{r=0}^{n} \frac{(q)_{n-r}}{(q)_{n}} \sum_{\substack{U \subseteq R^{n} \\
\nu\left(R^{n} / U\right)=r}}\left[R^{n}: U\right]^{-(n+u)} \quad \text { by } 2.3,2.4 \\
& =\mathcal{S}_{\leq n}(R ; u) \\
& \leq \frac{1}{(q)_{n}} \sum_{r=0}^{n} \sum_{\substack{U \subseteq R^{n} \\
\nu\left(R^{n} / U\right)=r}}\left[R^{n}: U\right]^{-(n+u)} \\
& =\frac{1}{(q)_{n}} \zeta_{R^{n}}(n+u),
\end{aligned}
$$

and the convergence of the sequence $\left(\frac{1}{(q)_{n}}\right)_{n \in \mathbb{N}}$.

b) We define the following abbreviation:

$$
\gamma_{u}(r, n):=\sum_{\substack{U \subseteq R^{n} \\ \nu\left(R^{n} / U\right)=r}}\left[R^{n}: U\right]^{-(n+u)} .
$$

We have to prove that the sequence

$$
\left(\mathcal{S}_{\leq n}(R ; u)-\zeta_{R^{n}}(n+u)\right)_{n \in \mathbb{N}}=\left(\sum_{r=0}^{n}\left(\frac{(q)_{n-r}}{(q)_{n}}-1\right) \gamma_{u}(r, n)\right)_{n \in \mathbb{N}}
$$

tends to zero. It is easy to see that

$$
1-\frac{(q)_{n}}{(q)_{n-r}} \leq q^{n-r+1}+q^{n-r+2}+\cdots+q^{n} \leq \frac{q^{n-r+1}}{1-q} .
$$

Hence

$$
\begin{aligned}
\sum_{r=0}^{n}\left(\frac{(q)_{n-r}}{(q)_{n}}-1\right) \gamma_{u}(r, n) & =\sum_{r=0}^{n} \frac{(q)_{n-r}}{(q)_{n}}\left(1-\frac{(q)_{n}}{(q)_{n-r}}\right) \gamma_{u}(r, n) \\
& \leq \frac{q^{n+1}}{(q)_{n}(1-q)} \sum_{r=0}^{n} p^{r} \gamma_{u}(r, n) .
\end{aligned}
$$

Now the claim follows if we can prove:

$$
\left(\sum_{r=0}^{n} p^{r} \gamma_{u}(r, n)\right)_{n \in \mathbb{N}} \text { is a bounded sequence. }
$$


Since $\nu\left(R^{n} / U\right)=\operatorname{dim}\left(R^{n} /\left(U+J^{n}\right)\right)$ we get

$$
\sum_{r=0}^{n} p^{r} \gamma_{u}(r, n)=\sum_{U \subseteq R^{n}}\left[R^{n}: U+J^{n}\right]\left[R^{n}: U\right]^{-(n+u)} \leq \zeta_{R^{n}}(n+u-1),
$$

and (4) follows from the assumption.

Sometimes it may be desirable to sum only over modules in certain isomorphism classes instead of computing the entire Cohen-Lenstra sum as in Definition 2.1. We will make use of this generalization in section 5. The following corollary is immediate.

Corollary 2.7. Let $\mathcal{M}$ be a set of non-isomorphic finite $R$-modules. If the sequence $\left(\zeta_{R^{n}}(n+u-1)\right)_{n \in \mathbb{N}}$ is bounded, then

$$
\sum_{M \in \mathcal{M}}\left|\operatorname{Aut}_{R}(M)\right|^{-1}|M|^{-u}=\lim _{n \rightarrow \infty} \sum_{M \in \mathcal{M}} \sum_{\substack{U \subseteq R^{n} \\ R^{n} / U \cong M}}\left[R^{n}: U\right]^{-(n+u)} .
$$

\section{The zeta function of a submodule of $\mathbb{Z}_{\mathbf{p}}\left[\mathbf{C}_{\mathbf{p}^{\mathrm{k}}}\right]^{\mathrm{n}}$ at $\mathbf{s}=\mathbf{n}$}

For $k \in \mathbb{N}$ put $R_{k}:=\mathbb{Z}_{p}\left[C_{p^{k}}\right]$, where $C_{p^{k}}$ is the multiplicative cyclic group of order $p^{k}$. Our goal in the next section will be to compute the Cohen-Lenstra sum $\mathcal{S}\left(R_{k} ; u\right)$ for $u \in \mathbb{N}$, along the lines of Theorem 2.6. We therefore have to study the zeta function of $R_{k}^{n}$ at $s=n$, as well as the zeta function of certain submodules of $R_{k}^{n}$ at $s=n$, as we will see in section 4 .

To this end we will use the main theorem of [14]. Let $\sigma$ be a generator of $C_{p^{k}}$, and set

$$
\phi_{k}=\sigma^{p^{k-1}(p-1)}+\sigma^{p^{k-1}(p-2)}+\cdots+\sigma^{p^{k-1}}+1 \in R_{k} .
$$

We assume $k>0$ and let

$$
f: R_{k}^{n} \rightarrow R_{k-1}^{n}
$$

be the canonical surjection, induced by the surjective homomorphism $\mathbb{Z}_{p}\left[C_{p^{k}}\right] \rightarrow \mathbb{Z}_{p}\left[C_{p^{k-1}}\right]$, mapping $\sigma$ to a fixed generator of $C_{p^{k-1}}$.

Theorem 3.1. Let $V \subseteq R_{k}^{n}$ be an $R_{k}$-submodule of finite index in $R_{k}^{n}$. Then the following formula holds for $s \in \mathbb{R}$ with $s>n-1$ :

$$
\zeta_{V}(s)=\prod_{j=0}^{n-1}\left(1-p^{j-s}\right)^{-1} \sum_{\bar{N} \subseteq V^{\circ}} p^{\left(n p^{k-1}-e_{V^{\circ}}(\bar{N})\right)(n-s)}[\bar{N}+f(V): \bar{N}]^{-s},
$$

where $V^{\circ}$ is given by $p V^{\circ}=f\left(V \cap \phi_{k} R_{k}^{n}\right)$ and $e_{V^{\circ}}(\bar{N})=\operatorname{dim}_{\mathbb{F}_{p}}(\bar{N}+$ $\left.p V^{\circ} / p V^{\circ}\right)$.

This is proved in [14, Th. 3.8, 3.9]. Note that $f$ maps $\phi_{k} R_{k}^{n}$ onto $p R_{k-1}^{n}$, hence $f\left(V \cap \phi_{k} R_{k}^{n}\right) \subseteq p R_{k-1}^{n}$. The fact that the zeta function of $V$ is defined for all $s \in \mathbb{R}$ with $s>n-1$ is a consequence of Solomon's First Conjecture 
proved in [1], and also follows in a more elementary way from the results in $[14$, Sec. 5].

If we consider formula (5) with $s=n$, it becomes much nicer:

$$
\zeta_{V}(n)=\frac{1}{(q)_{n}} \sum_{\bar{N} \subseteq V^{\circ}}[\bar{N}+f(V): \bar{N}]^{-n},
$$

where again $V \subseteq R_{k}^{n}$ is a submodule of finite index.

Theorem 3.2. The zeta function of $R_{k}^{n}$ at $s=n$ equals $\zeta_{R_{k}^{n}}(n)=\frac{1}{(q)_{n}^{k+1}}$.

Proof. We proceed by induction on $k$. If $k=0$ the result follows from the well-known formula

$$
\zeta_{\mathbb{Z}_{p}^{n}}(s)=\prod_{j=0}^{n-1}\left(1-p^{j-s}\right)^{-1},
$$

cf. [14, Th. 3.9]. If $k>0$ then obviously $\left(R_{k}^{n}\right)^{\circ}=R_{k-1}^{n}$, and (6) yields

$$
\zeta_{R_{k}^{n}}(n)=\frac{1}{(q)_{n}} \sum_{\bar{N} \subseteq R_{k-1}^{n}}\left[R_{k-1}^{n}: \bar{N}\right]^{-n}=\frac{1}{(q)_{n}} \zeta_{R_{k-1}^{n}}(n),
$$

whence the claim follows.

Using the concept of a Möbius function, we can find a more appropriate expression for (6). Thus let again $V \subseteq R_{k}^{n}$ be a submodule of finite index, and let $\mu$ be the Möbius function (cf. [11]) of the lattice of submodules of $V^{\circ}$ having finite index in $V^{\circ}$.

\section{Lemma 3.3.}

$$
\zeta_{V}(n)=\frac{1}{(q)_{n}} \sum_{f(V) \subseteq \bar{Y} \subseteq V^{\circ}}\left(\sum_{\bar{Y} \subseteq \bar{W} \subseteq V^{\circ}} \mu(\bar{Y}, \bar{W})[\bar{W}: \bar{Y}]^{-n}\right) \zeta_{\bar{Y}}(n),
$$

where $f(V)$ and $V^{\circ}$ are defined as in Theorem 3.1.

Proof. We have

$$
\zeta_{V}(n)=\frac{1}{(q)_{n}} \sum_{f(V) \subseteq \bar{W} \subseteq V^{\circ}} \eta(\bar{W}),
$$

where for $f(V) \subseteq \bar{Y} \subseteq V^{\circ}$ we set

$$
\eta(\bar{Y}):=\sum_{\substack{\bar{N} \subseteq \bar{Y} \\ \bar{N}+f(V)=\bar{Y}}}[\bar{Y}: \bar{N}]^{-n} .
$$

One easily verifies that

$$
\sum_{f(V) \subseteq \bar{Y} \subseteq \bar{W}}[\bar{W}: \bar{Y}]^{-n} \eta(\bar{Y})=\zeta_{\bar{W}}(n)
$$


(this is analogous to the proof of Theorem 4.5 in [14]). Applying the Möbius inversion formula [11, Sec. 3, Prop. 2] yields

$$
\zeta_{V}(n)=\frac{1}{(q)_{n}} \sum_{f(V) \subseteq \bar{W} \subseteq V^{\circ}} \sum_{f(V) \subseteq \bar{Y} \subseteq \bar{W}} \mu(\bar{Y}, \bar{W})[\bar{W}: \bar{Y}]^{-n} \zeta_{\bar{Y}}(n),
$$

and the formula stated above follows.

For the rest of this section, we let $R=R_{k}$ and $\bar{R}=R_{k-1}$. Let $J, \bar{J}$ the maximal ideals of $R, \bar{R}$ respectively. We will use the above lemma to derive a formula for $\zeta_{V}(n)$, where $V$ is an $R$-module such that $J^{n} \subseteq V \subseteq R^{n}$.

Lemma 3.4. Let $J^{n} \subseteq V \subseteq R^{n}$ be a submodule. Then $\bar{J}^{n} \subseteq f(V) \subseteq \bar{R}^{n}$, and

$$
\zeta_{V}(n)=\sum_{f_{2}(V) \subseteq \bar{Y} \subseteq \bar{R}^{n}} \frac{1}{(q)_{j(\bar{Y})}} \zeta_{\bar{Y}}(n),
$$

where $j(\bar{Y}):=\operatorname{dim}_{\mathbb{F}_{p}}\left(\bar{Y} / \bar{J}^{n}\right)$.

Proof. Clearly $f\left(J^{n}\right)=\bar{J}^{n}$, so $\bar{J}^{n} \subseteq f(V) \subseteq \bar{R}^{n}$. Since $\phi_{k} \in J$ we have

$$
p V^{\circ}=f\left(V \cap \phi_{k} R^{n}\right) \supseteq f\left(J^{n} \cap \phi_{k} R^{n}\right)=f\left(\phi_{k} R^{n}\right)=p \bar{R}^{n},
$$

thus $V^{\circ}=\bar{R}^{n}$. The preceding lemma implies

$$
\zeta_{V}(n)=\frac{1}{(q)_{n}} \sum_{f(V) \subseteq \bar{Y} \subseteq \bar{R}^{n}}\left(\sum_{\bar{Y} \subseteq \bar{W} \subseteq \bar{R}^{n}} \mu(\bar{Y}, \bar{W})[\bar{W}: \bar{Y}]^{-n}\right) \zeta_{\bar{Y}}(n) .
$$

Fix a submodule $\bar{Y}$ such that $\bar{J}^{n} \subseteq \bar{Y} \subseteq \bar{R}^{n}$, and put $j:=j(\bar{Y})$. Then the lattice of $\bar{R}$-submodules of $\bar{R}^{n}$ containing $\bar{Y}$ is isomorphic to the lattice of

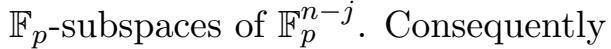

$$
\sum_{\bar{Y} \subseteq \bar{W} \subseteq \bar{R}^{n}} \mu(\bar{Y}, \bar{W})[\bar{W}: \bar{Y}]^{-n}=\sum_{U \subseteq \mathbb{F}_{p}^{n-j}} \widetilde{\mu}(0, U)|U|^{-n},
$$

where $\widetilde{\mu}$ is the Möbius function of the lattice of subspaces of $\mathbb{F}_{p}^{n-j}$. Since

$$
\widetilde{\mu}(0, U)=(-1)^{\operatorname{dim}(U)} p^{(\underset{2}{\operatorname{dim}(U)})}
$$

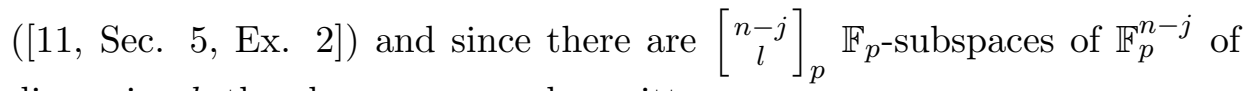
dimension $l$, the above sum can be written as

$$
\sum_{l=0}^{n-j}\left[\begin{array}{c}
n-j \\
l
\end{array}\right]_{p}(-1)^{l} p^{\left(\begin{array}{l}
l \\
2
\end{array}\right) p^{-l n}=\prod_{i=0}^{n-j-1}\left(1-p^{i-n}\right)=\frac{(q)_{n}}{(q)_{j}},}
$$

where the equality of the sum and the product follows from [8, III.8.5]. Putting together this result with (9) proves the lemma. 
Using an inductive argument, the lemma shows in particular that the value $\zeta_{V}(n)$ only depends on the $\mathbb{F}_{p}$-dimension of $V / J^{n}$, i.e.

$$
\zeta_{V}(n)=\zeta_{V^{\prime}}(n) \quad \text { if } \quad \operatorname{dim}_{\mathbb{F}_{p}}\left(V / J^{n}\right)=\operatorname{dim}_{\mathbb{F}_{p}}\left(V^{\prime} / J^{n}\right) .
$$

Notation. Let $0 \leq m \leq n$. We define

$c_{k}^{n}(m):=\zeta_{V}(n) \quad$ for any $J^{n} \subseteq V \subseteq R^{n}$ with $\operatorname{dim}_{\mathbb{F}_{p}}\left(V / J^{n}\right)=m$.

If $k=0$ we have $V \cong \mathbb{Z}_{p}^{n}$, hence by (7)

$$
c_{0}^{n}(m)=\frac{1}{(q)_{n}} \quad \forall 0 \leq m \leq n .
$$

If $k>0$ the equality $\left[V: J^{n}\right]=\left[f(V): \bar{J}^{n}\right]$, together with the preceding lemma, implies

$$
c_{k}^{n}(m)=\sum_{j=m}^{n}\left[\begin{array}{l}
n-m \\
j-m
\end{array}\right]_{p} \frac{c_{k-1}^{n}(j)}{(q)_{j}},
$$

and this recursion formula allows the explicit computation of $\zeta_{V}(n)$. For example, if $k=1$, i.e. $R=\mathbb{Z}_{p}\left[C_{p}\right]$ and $J=\operatorname{rad}(R)$, we get

$$
\zeta_{J^{n}}(n)=c_{1}^{n}(0)=\frac{1}{(q)_{n}} \sum_{j=0}^{n}\left[\begin{array}{l}
n \\
j
\end{array}\right]_{p} \frac{1}{(q)_{j}} .
$$

\section{Cohen-Lenstra sums over $\mathbb{Z}_{\mathbf{p}}\left[\mathbf{C}_{\mathbf{p}^{\mathbf{k}}}\right]$}

In this section we want to evaluate the Cohen-Lenstra sums $\mathcal{S}\left(\mathbb{Z}_{p}\left[C_{p^{k}}\right] ; u\right)$, where $u \in \mathbb{N}$ and $C_{p^{k}}$ is the multiplicative cyclic group of order $p^{k}$. We put

$$
R=\mathbb{Z}_{p}\left[C_{p^{k}}\right]
$$

By Theorem 3.2 the sequence $\left(\zeta_{R^{n}}(n)\right)_{n \in \mathbb{N}}$ is convergent, and thus

$$
\mathcal{S}(R ; u)=\lim _{n \rightarrow \infty} \zeta_{R^{n}}(n+u) \in \mathbb{R}_{+} \quad \forall u \geq 1
$$

according to Theorem 2.6. Note that the explicit formulas in [14] for $\zeta_{R^{n}}(s)$ in the cases $k=1,2$ are useful for approximating the value of $\mathcal{S}(R ; u)$.

It remains to determine

$$
S(R ; 0)=\sum_{M}\left|\operatorname{Aut}_{R}(M)\right|^{-1} .
$$

Since the zeta function $\zeta_{R^{n}}(s)$ is not defined for $s=n-1$, Theorem 2.6 is not applicable. So first of all it is interesting to investigate whether $S(R ; 0)$ converges to real number. This question was asked by Greither in [5], and he conjectured that $S(R ; 0)$ converges to $(q)_{\infty}^{-(k+1)}$. We will prove this conjecture in Corollary 4.3 below. 
Theorem 4.1. Let $R=\mathbb{Z}_{p}\left[C_{p^{k}}\right]$. Then

$$
\mathcal{S}(R ; 0)=\lim _{n \rightarrow \infty} \zeta_{R^{n}}(n) .
$$

Proof. Let $\gamma_{0}(r, n)$ be defined as in (3). Following the steps in the proof of Theorem 2.6, it remains to show the assertion (4):

$$
\left(\sum_{r=0}^{n} p^{r} \gamma_{0}(r, n)\right)_{n \in \mathbb{N}} \text { is a bounded sequence. }
$$

One has

$$
\begin{aligned}
\gamma_{0}(r, n) & =\sum_{\substack{U \subseteq R^{n} \\
\operatorname{dim}\left(R^{n} /\left(U+J^{n}\right)\right)=r}}\left[R^{n}: U\right]^{-n} \\
& \leq q^{r n} \sum_{\substack{J^{n} \subseteq V \subseteq R^{n} \\
\operatorname{dim}\left(R^{n} / V\right)=r}} \zeta_{V}(n) .
\end{aligned}
$$

In the preceding section we saw that $\zeta_{V}(n)$ only depends on $\operatorname{dim}\left(V / J^{n}\right)=$ $n-r$, so using the notation introduced in (10) we get

$$
\gamma_{0}(r, n) \leq q^{r n}\left[\begin{array}{l}
n \\
r
\end{array}\right]_{p} c_{k}^{n}(n-r) \leq \frac{q^{r^{2}}}{(q)_{r}} c_{k}^{n}(n-r) .
$$

The next lemma shows that there exists a constant $A>0$, independent of $r$ and $n$, such that

$$
\sum_{r=0}^{n} p^{r} \gamma_{0}(r, n) \leq \sum_{r=0}^{n} p^{r} \frac{q^{r^{2}}}{(q)_{r}} \cdot A \cdot p^{r(r+2) / 2} \leq \frac{A}{(q)_{\infty}} \sum_{r=0}^{\infty} q^{\left(r^{2}-4 r\right) / 2}
$$

whence the theorem is proved.

Lemma 4.2. For all $k \in \mathbb{N}$ there exists a constant $A>0$, independent of $n$ and $0 \leq r \leq n$, such that the values $c_{k}^{n}(n-r)$ defined in (10) satisfy the inequality

$$
c_{k}^{n}(n-r) \leq A \cdot p^{r(r+2) / 2} .
$$

Proof. We proceed by induction on $k$. If $k=0$ we can simply set $A:=(q)_{\infty}^{-1}$ by (11). Let $k>0$, and let $A^{\prime}>0$ be a constant satisfying

$$
c_{k-1}^{n}(n-l) \leq A^{\prime} \cdot p^{l(l+2) / 2}
$$


for all $n$ and all $0 \leq l \leq n$. For $n \in \mathbb{N}$ and $0 \leq r \leq n$, the recursion formula (12) implies

$$
\begin{aligned}
c_{k}^{n}(n-r) & =\sum_{j=n-r}^{n}\left[\begin{array}{c}
r \\
j-(n-r)
\end{array}\right]_{p} \frac{c_{k-1}^{n}(j)}{(q)_{j}} \\
& \leq \frac{A^{\prime}}{(q)_{n}} \sum_{i=0}^{r}\left[\begin{array}{l}
r \\
i
\end{array}\right]_{p} p^{(r-i)(r-i+2) / 2} \\
& \leq \frac{A^{\prime}}{(q)_{n}(q)_{r}} \sum_{i=0}^{r} p^{i(r-i)} p^{(r-i)(r-i+2) / 2} \\
& =\frac{A^{\prime}}{(q)_{n}(q)_{r}} p^{r(r+2) / 2} \sum_{i=0}^{r} p^{-i(i+2) / 2} .
\end{aligned}
$$

Therefore we can put

$$
A:=\frac{A^{\prime}}{(q)_{\infty}^{2}} \sum_{i=0}^{\infty} q^{i(i+2) / 2}
$$

We remark that Corollary 2.7 holds for $R=\mathbb{Z}_{p}\left[C_{p^{k}}\right]$ and $u=0$ as well: If $\mathcal{M}$ is a set of non-isomorphic finite $R$-modules, then

$$
\sum_{M \in \mathcal{M}}\left|\operatorname{Aut}_{R}(M)\right|^{-1}=\lim _{n \rightarrow \infty} \sum_{M \in \mathcal{M}} \sum_{\substack{U \subseteq R^{n} \\ R^{n} / U \cong M}}\left[R^{n}: U\right]^{-n} .
$$

Now Greither's conjecture (cf. [5]) is a direct consequence of Theorem 4.1 and 3.2 .

Corollary 4.3. The Cohen-Lenstra sum $S\left(\mathbb{Z}_{p}\left[C_{p^{k}}\right] ; 0\right)$ converges to a real number. More precisely: $\mathcal{S}\left(\mathbb{Z}_{p}\left[C_{p^{k}}\right] ; 0\right)=\frac{1}{(q)_{\infty}^{k+1}}$.

\section{Cohen-Lenstra sums over $\mathbb{Z}_{\mathbf{p}}\left[\mathbf{C}_{\mathbf{p}}\right]$ with prescribed cohomology groups}

In this section we will consider some "refinements" of Cohen-Lenstra sums over the ring $\mathbb{Z}_{p}\left[C_{p}\right]$. To be more precise, we will restrict the summation to those finite modules $M$ having prescribed Tate cohomology groups $\widehat{H}^{i}\left(C_{p}, M\right)$. Sums of this kind may be important for applications; e.g. in $[5]$

$$
\sum_{M}\left|\operatorname{Aut}_{\mathbb{Z}_{p}[\Delta]}(M)\right|^{-1}
$$

is computed, where $\Delta$ is a finite abelian $p$-group, and the summation extends over all cohomologically trivial $\mathbb{Z}_{p}[\Delta]$-modules. 
We use the following notations in this section. Let $R=\mathbb{Z}_{p}\left[C_{p}\right]$, let $\sigma$ be a generator of the cyclic group $C_{p}$, and put $\phi=1+\sigma+\cdots+\sigma^{p-1} \in R$ and $I=(\sigma-1) R$ (which is the augmentation ideal of $R$ ).

We need some basic notions of Tate cohomology of finite groups (cf. [12]). If $M$ is a finite $R$-module, the Tate cohomology groups satisfy

$$
\widehat{H}^{i}\left(C_{p}, M\right) \cong \widehat{H}^{i+2}\left(C_{p}, M\right) \quad \forall i \in \mathbb{Z},
$$

for $C_{p}$ is cyclic. Hence we can restrict to

$$
\widehat{H}^{0}\left(C_{p}, M\right)=M^{C_{p}} / \phi M \text { and } \widehat{H}^{1}\left(C_{p}, M\right) \cong \widehat{H}^{-1}\left(C_{p}, M\right)={ }_{\phi} M / I M ;
$$

here $M^{C_{p}}$ is the submodule of elements fixed by $C_{p}$, and ${ }_{\phi} M$ is the kernel of the action of $\phi$ on $M$. Since $M$ is finite, its Herbrand quotient is equal to 1 , i.e. $\left|\widehat{H}^{0}\left(C_{p}, M\right)\right|=\left|\widehat{H}^{1}\left(C_{p}, M\right)\right|$. Since all cohomology groups are annihilated by $\left|C_{p}\right|$, we infer that there exists $h \in \mathbb{N}$ such that

$$
\widehat{H}^{0}\left(C_{p}, M\right) \cong \widehat{H}^{1}\left(C_{p}, M\right) \cong(\mathbb{Z} / p \mathbb{Z})^{h} .
$$

This number $h$ describes completely all Tate cohomology groups $\widehat{H}^{i}\left(C_{p}, M\right)$. We will use the following abbreviation:

$$
\widehat{H}^{i}(M):=\widehat{H}^{i}\left(C_{p}, M\right)
$$

for $i=0,1$.

Now let $G$ be a finite abelian $p$-group and $h, u \in \mathbb{N}$. The goal of this section is the computation of

$$
\sum_{\substack{\phi M \cong G \\\left|\hat{H}^{1}(M)\right|=p^{h}}}\left|\operatorname{Aut}_{R}(M)\right|^{-1}|M|^{-u},
$$

where of course the summation extends over all finite modules $M$ as indicated, up to isomorphism. Note that $\phi M$ is an $(R / I)$-module, and $R / I \cong \mathbb{Z}_{p}$.

The value of this sum will be stated in Theorem 5.6. A first step in the computation consists in relating this sum over finite modules $M$ to a limit for $n \rightarrow \infty$ of a sum over submodules $U \subseteq R^{n}$ (a kind of "partial zeta function"), similar to the case of the full Cohen-Lenstra sum in section 2.

We denote by $\varepsilon: R^{n} \rightarrow \mathbb{Z}_{p}^{n}$ the augmentation map with kernel $I^{n}$, induced by $R \rightarrow \mathbb{Z}_{p}, \sum_{i=0}^{p-1} a_{i} \sigma^{i} \mapsto \sum_{i=0}^{p-1} a_{i}$, and by $\nu:=\nu(G)=\operatorname{dim}_{\mathbb{F}_{p}}(G / p G)$ the rank of the finite abelian $p$-group $G$. We further recall that all submodules of $R^{n}$ are understood to have finite index in $R^{n}$. 
Lemma 5.1. Let $G$ be a finite abelian p-group, and $h, u \in \mathbb{N}$. Then for all $N \subseteq R^{n}$ there is $\bar{N} \subseteq \mathbb{Z}_{p}^{n}$ such that $p \bar{N}=\varepsilon\left(N \cap \phi R^{n}\right)$, and

$$
\sum_{\substack{\phi M \cong G \\\left|\hat{H}^{1}(M)\right|=p^{h}}}\left|\operatorname{Aut}_{R}(M)\right|^{-1}|M|^{-u}=\lim _{n \rightarrow \infty} \sum_{\substack{N \subseteq R^{n} \\ \mathbb{Z}_{p}^{n} / \bar{N} \cong G \\[N: \varepsilon(N)]=p^{h}}}\left[R^{n}: N\right]^{-(n+u)} .
$$

Proof. The existence of $\bar{N}$ is clear. Multiplication by $\phi$ on $M$ induces a surjection $\psi: M / I M \rightarrow \phi M$ with $\widehat{H}^{1}(M)=\operatorname{ker}(\psi)$. Each $M$ such that $\phi M \cong G$ and $\left|\widehat{H}^{1}(M)\right|=p^{h}$ has the form $M \cong R^{n} / N$ for some $n \geq \max \{\nu, h\}$ and $N \subseteq R^{n}$. Thus

$$
M / I M \cong R^{n} /\left(N+I^{n}\right) \cong \mathbb{Z}_{p}^{n} / \varepsilon(N)
$$

and

$$
\phi M \cong\left(\phi R^{n}+N\right) / N \cong \phi R^{n} /\left(N \cap \phi R^{n}\right) \cong p \mathbb{Z}_{p}^{n} / \varepsilon\left(N \cap \phi R^{n}\right) \cong \mathbb{Z}_{p}^{n} / \bar{N} .
$$

We therefore have a commutative diagram

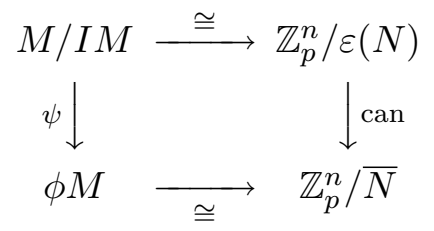

hence

$$
\widehat{H}^{1}(M)=\operatorname{ker}(\psi) \cong \bar{N} / \varepsilon(N) .
$$

Now the lemma follows from Theorem 4.1, or more precisely from its generalization stated at the end of the preceding section.

We now have to determine all $N \subseteq R^{n}$ such that $\mathbb{Z}_{p}^{n} / \bar{N} \cong G$ and $[\bar{N}: \varepsilon(N)]=p^{h}$. In order to achieve this, we will use Morita's Theorem (cf. [9, Sec. 3.12]) and translate all submodules of $R^{n}$ to left ideals of the matrix ring $\mathrm{M}_{n}(R)$. The main property of Morita's Theorem that we will be using in the sequel is the following: There is an isomorphism between the lattice of $R$-submodules $U$ of finite index in $R^{n}$ and the lattice of left ideals $I \subseteq \mathrm{M}_{n}(R)$ of finite index. Moreover, if $U$ and $I$ correspond to each other, then one easily verifies that

$$
\left[\mathrm{M}_{n}(R): I\right]=\left[R^{n}: U\right]^{n} .
$$

In a similar way, submodules of $\mathbb{Z}_{p}^{n}$ correspond to left ideals of $\mathrm{M}_{n}\left(\mathbb{Z}_{p}\right)$.

Let $n \geq \max \{\nu, h\}$. Then $G$ is a quotient of $\mathbb{Z}_{p}^{n}$, and we let $G^{\prime}$ be the corresponding quotient of $\mathrm{M}_{n}\left(\mathbb{Z}_{p}\right)$ via Morita's Theorem, so in particular

$$
\left|G^{\prime}\right|=|G|^{n} .
$$


Now it is easy to see from the above lemma that our sum is equal to the limit for $n \rightarrow \infty$ of

$$
x_{n}:=\sum_{\substack{N^{\prime} \subseteq \mathrm{M}_{n}(R) \\ \mathrm{M}_{n}\left(\mathbb{Z}_{p}\right) / N^{\prime} \cong G^{\prime} \\\left[N^{\prime}: \varepsilon\left(N^{\prime}\right)\right]=p^{n h}}}\left[\mathrm{M}_{n}(R): N^{\prime}\right]^{-(1+u / n)},
$$

where as always all ideals are of finite index, and $\overline{N^{\prime}}$ is the left ideal of $\mathrm{M}_{n}\left(\mathbb{Z}_{p}\right)$ satisfying $p \overline{N^{\prime}}=\varepsilon\left(N^{\prime} \cap \phi \mathrm{M}_{n}(R)\right)$. Here we denote the augmentation $\operatorname{map} \mathrm{M}_{n}(R) \rightarrow \mathrm{M}_{n}\left(\mathbb{Z}_{p}\right)$ by $\varepsilon$ as well.

Thus we have to count left ideals of $\mathrm{M}_{n}(R)$. This can be done by using an idea that goes back to Reiner (cf. [10]), also applied in [14, Sec. 3]. The crucial point is that $R=\mathbb{Z}_{p}\left[C_{p}\right]$ is a fibre product of the two discrete valuation rings $S=\mathbb{Z}_{p}[\omega]$, where $\omega$ is a primitive $p$-th root of unity, and $\mathbb{Z}_{p}$. This leads to a fibre product representation for $\mathrm{M}_{n}(R)$, viz there is a fibre product diagram with surjective maps

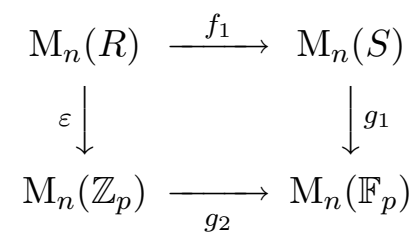

with $f_{1}$ induced by $R \rightarrow R /(\phi) \cong S, g_{1}$ induced by $S \rightarrow S /(1-\omega) \cong \mathbb{F}_{p}$, and $g_{2}$ is reduction mod $p$. Equivalently, there is an isomorphism

$$
\mathrm{M}_{n}(R) \cong\left\{(x, y) \in \mathrm{M}_{n}(S) \times \mathrm{M}_{n}\left(\mathbb{Z}_{p}\right) \mid g_{1}(x)=g_{2}(y)\right\} .
$$

Now we can use Reiner's method, and represent the left ideals of $\mathrm{M}_{n}(R)$ in terms of the left ideals of $\mathrm{M}_{n}(S)$ and $\mathrm{M}_{n}\left(\mathbb{Z}_{p}\right)$ (both of which are principal ideal rings). If $N^{\prime} \subseteq \mathrm{M}_{n}(R)$ is a left ideal (of finite index), then there is an $\alpha \in \mathrm{M}_{n}(S)$ with $\operatorname{det}(\alpha) \neq 0$ such that $f_{1}\left(N^{\prime}\right)=\mathrm{M}_{n}(S) \alpha$. Choose $\beta \in \mathrm{M}_{n}\left(\mathbb{Z}_{p}\right)$ such that $g_{1}(\alpha)=g_{2}(\beta)$. Then

$$
N^{\prime}=\mathrm{M}_{n}(R)(\alpha, \beta)+\left(0, p \overline{N^{\prime}}\right),
$$

where $\overline{N^{\prime}} \subseteq \mathrm{M}_{n}\left(\mathbb{Z}_{p}\right)$ is the left ideal (of finite index) satisfying $p \overline{N^{\prime}}=$ $\varepsilon\left(N^{\prime} \cap \phi \mathrm{M}_{n}(R)\right)=\left\{x \in \mathrm{M}_{n}\left(\mathbb{Z}_{p}\right) \mid(0, x) \in N^{\prime}\right\}$, and $\beta \in \overline{N^{\prime}}$.

Conversely, if $\alpha \in \mathrm{M}_{n}(S)$ with $\operatorname{det}(\alpha) \neq 0$ and a left ideal $\overline{N^{\prime}} \subseteq \mathrm{M}_{n}\left(\mathbb{Z}_{p}\right)$ of finite index are given, then $\alpha$ and $\overline{N^{\prime}}$ give rise to a left ideal $N^{\prime} \subseteq \mathrm{M}_{n}(R)$ as in (13) if and only if $g_{1}(\alpha) \in g_{2}\left(\overline{N^{\prime}}\right)$. In this case, the number of left ideals of $\mathrm{M}_{n}(R)$ belonging to $\alpha$ and $\overline{N^{\prime}}$ is equal to the number of $\beta \in \overline{N^{\prime}}$ distinct $\bmod p \overline{N^{\prime}}$ such that $g_{1}(\alpha)=g_{2}(\beta)$.

Notation. We denote by $\mathcal{R}$ a system of representatives of the generators of all left ideals of finite index in $\mathrm{M}_{n}(S)$. If $\alpha \in \mathcal{R}$ and $\overline{N^{\prime}} \subseteq \mathrm{M}_{n}\left(\mathbb{Z}_{p}\right)$ 
is a left ideal with $g_{1}(\alpha) \in g_{2}\left(\overline{N^{\prime}}\right)$ we denote by $\theta(\alpha)$ the number of left $\mathrm{M}_{n}(R)$-ideals of the form

$$
N^{\prime}:=\mathrm{M}_{n}(R)(\alpha, \beta)+\left(0, p \overline{N^{\prime}}\right)
$$

satisfying $\left[\overline{N^{\prime}}: \mathrm{M}_{n}\left(\mathbb{Z}_{p}\right) \beta+p \overline{N^{\prime}}\right]=p^{n h}$. Note that the latter is one of the conditions required in the summation for $x_{n}$, since $\varepsilon\left(N^{\prime}\right)=\mathrm{M}_{n}\left(\mathbb{Z}_{p}\right) \beta+p \overline{N^{\prime}}$. We will see below in Lemma 5.3 that the value $\theta(\alpha)$ does not depend on the particular $\overline{N^{\prime}}$, which justifies the notation.

It is shown in [14, Lemma 3.4] that

$$
\left[\mathrm{M}_{n}(R): N^{\prime}\right]=\left[\mathrm{M}_{n}(S): \mathrm{M}_{n}(S) \alpha\right]\left[\mathrm{M}_{n}\left(\mathbb{Z}_{p}\right): \overline{N^{\prime}}\right]
$$

for $N^{\prime}$ as in (13). Together with the above discussion, this equality yields the following formula for $x_{n}$ :

$$
x_{n}=\sum_{\substack{\overline{N^{\prime}} \subseteq \mathrm{M}_{n}\left(\mathbb{Z}_{p}\right) \\ \mathrm{M}_{n}\left(\mathbb{Z}_{p}\right) / \overline{N^{\prime}} \cong G^{\prime}}} \sum_{\substack{\alpha \in \mathcal{R} \\ \alpha \in g_{1}^{-1}\left(g_{2}\left(\overline{N^{\prime}}\right)\right)}} \theta(\alpha)\left(\left[\mathrm{M}_{n}(S): \mathrm{M}_{n}(S) \alpha\right]\left[\mathrm{M}_{n}\left(\mathbb{Z}_{p}\right): \overline{N^{\prime}}\right]\right)^{-(1+u / n)}
$$

hence $x_{n}=y_{n} z_{n}$ with

$$
\begin{aligned}
& y_{n}:=\sum_{\substack{\overline{N^{\prime}} \subseteq \mathrm{M}_{n}\left(\mathbb{Z}_{p}\right) \\
\mathrm{M}_{n}\left(\mathbb{Z}_{p}\right) / \overline{N^{\prime}} \cong G^{\prime}}}\left|G^{\prime}\right|^{-(1+u / n)}, \\
& z_{n}:=\sum_{\substack{\alpha \in \mathcal{R} \\
g_{1}(\alpha) \in g_{2}\left(\overline{N^{\prime}}\right)}} \theta(\alpha)\left[\mathrm{M}_{n}(S): \mathrm{M}_{n}(S) \alpha\right]^{-(1+u / n)},
\end{aligned}
$$

where in the last sum $\overline{N^{\prime}} \subseteq \mathrm{M}_{n}\left(\mathbb{Z}_{p}\right)$ is an arbitrary left ideal with $\mathrm{M}_{n}\left(\mathbb{Z}_{p}\right) / \overline{N^{\prime}} \cong G^{\prime}$.

Lemma 5.2. $\lim _{n \rightarrow \infty} y_{n}=|\operatorname{Aut}(G)|^{-1}|G|^{-u}$.

Proof. We translate everything back to submodules of $\mathbb{Z}_{p}^{n}$ using Morita's Theorem. Since $\left|G^{\prime}\right|=|G|^{n}$ we get

$$
y_{n}=|G|^{-(n+u)} \cdot\left|\left\{\bar{N} \subseteq \mathbb{Z}_{p}^{n} \mid \mathbb{Z}_{p}^{n} / \bar{N} \cong G\right\}\right|,
$$

and by Lemma 2.2, 2.4 we infer

$$
y_{n}=|G|^{-(n+u)}|G|^{n} \frac{(q)_{n}}{(q)_{n-\nu}}|\operatorname{Aut}(G)|^{-1},
$$

which proves the claim.

The calculation of $\lim _{n \rightarrow \infty} z_{n}$ is more complicated. We start by computing $\theta(\alpha)$, and we recall that $\nu$ denotes the rank of the abelian $p$-group G. 
Lemma 5.3. Let $\overline{N^{\prime}} \subseteq \mathrm{M}_{n}\left(\mathbb{Z}_{p}\right)$ be a left ideal such that $\mathrm{M}_{n}\left(\mathbb{Z}_{p}\right) / \overline{N^{\prime}} \cong G^{\prime}$. Furthermore let $\alpha \in \mathcal{R}$ with $g_{1}(\alpha) \in g_{2}\left(\overline{N^{\prime}}\right)$, and put $r:=\operatorname{rk}\left(g_{1}(\alpha)\right)$. Then $\theta(\alpha)$ equals $\theta_{r}$, the number of all $\xi \in \mathrm{M}_{n}\left(\mathbb{F}_{p}\right)$ lying in

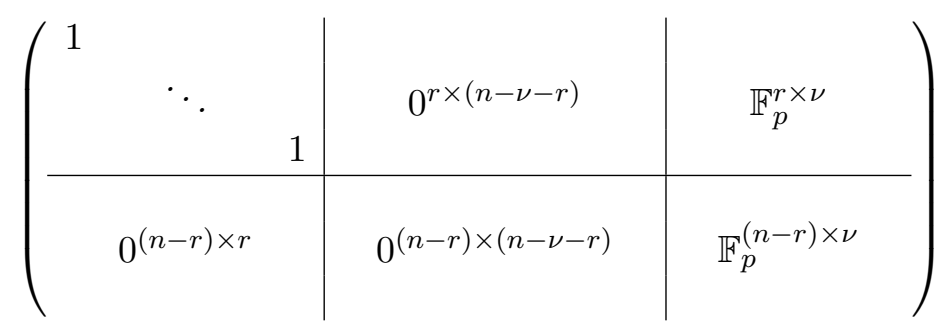

and whose bottom right $((n-r) \times \nu)$-submatrix has rank $n-h-r$. In particular we have

$$
n-\nu-h \leq r \leq \min \{n-\nu, n-h\} .
$$

Proof. Fix $\alpha$ and $\overline{N^{\prime}} \subseteq \mathrm{M}_{n}\left(\mathbb{Z}_{p}\right)$ as above. The number of left $\mathrm{M}_{n}(R)$-ideals of the form (13) equals the number of $\beta \in \overline{N^{\prime}}$ with $g_{1}(\alpha)=g_{2}(\beta)$ which are distinct $\bmod p \overline{N^{\prime}}$. Thus, by definition of $\theta(\alpha)$,

$\theta(\alpha)=\left|\left\{\beta \in \overline{N^{\prime}} \bmod p \overline{N^{\prime}} \mid g_{1}(\alpha)=g_{2}(\beta),\left[\overline{N^{\prime}}: \mathrm{M}_{n}\left(\mathbb{Z}_{p}\right) \beta+p \overline{N^{\prime}}\right]=p^{n h}\right\}\right|$.

Choose $\rho \in \mathrm{M}_{n}\left(\mathbb{Z}_{p}\right)$ with $\mathrm{M}_{n}\left(\mathbb{Z}_{p}\right) \rho=\overline{N^{\prime}}$. There is an isomorphism

$$
G^{\prime} / p G^{\prime} \cong \mathrm{M}_{n}\left(\mathbb{F}_{p}\right) / g_{2}\left(\overline{N^{\prime}}\right)=\mathrm{M}_{n}\left(\mathbb{F}_{p}\right) / \mathrm{M}_{n}\left(\mathbb{F}_{p}\right) g_{2}(\rho),
$$

whence $\operatorname{rk}\left(g_{2}(\rho)\right)=n-\nu$. Now $\theta(\alpha)$ equals the number of all $\beta^{\prime} \in$ $\mathrm{M}_{n}\left(\mathbb{Z}_{p}\right) \bmod p \mathrm{M}_{n}\left(\mathbb{Z}_{p}\right)$ such that

$$
g_{1}(\alpha)=g_{2}\left(\beta^{\prime}\right) g_{2}(\rho) \text { and }\left[\mathrm{M}_{n}\left(\mathbb{Z}_{p}\right) \beta^{\prime}+p \mathrm{M}_{n}\left(\mathbb{Z}_{p}\right): p \mathrm{M}_{n}\left(\mathbb{Z}_{p}\right)\right]=p^{n(n-h)} .
$$

We assume without loss of generality that

$$
g_{2}(\rho)=\left(\begin{array}{cccccc}
1 & & & & & \\
& \ddots & & & & \\
& & 1 & & & \\
& & & 0 & \ddots & \\
& & & & & 0
\end{array}\right)
$$

with $n-\nu 1$ 's on the main diagonal. Then

$$
g_{1}(\alpha) \in\left(\mathbb{F}_{p}^{n \times(n-\nu)} \mid 0^{n \times \nu}\right),
$$

i.e. $g_{1}(\alpha)=\left(\gamma_{1} \mid 0\right)$ for some $\gamma_{1} \in \mathbb{F}_{p}^{n \times(n-\nu)}$ with $\operatorname{rk}\left(\gamma_{1}\right)=r$. This implies

$$
\theta(\alpha)=\mid\left\{\xi=\left(\xi_{1} \mid \xi_{2}\right) \in\left(\mathbb{F}_{p}^{n \times(n-\nu)} \mid \mathbb{F}_{p}^{n \times \nu}\right) \mid \xi_{1}=\gamma_{1} \text { and } \operatorname{rk}(\xi)=n-h\right\} \mid .
$$

Obviously this number only depends on $r=\operatorname{rk}\left(\gamma_{1}\right)$. Therefore we may choose $\gamma_{1}$ to be the matrix having $r$ 1's as its first entries of the main diagonal, all other entries being 0 . Now it is clear that $\theta(\alpha)=\theta_{r}$. 
Since $g_{1}(\alpha) \in g_{2}\left(\overline{N^{\prime}}\right)$ we have $\theta_{r}=\theta(\alpha) \neq 0$, or equivalently $n-\nu-h \leq$ $r \leq \min \{n-\nu, n-h\}$.

The following lemma, which is easy to prove (cf. [4, Th. 2]) gives a formula for the number of matrices of given size over a finite field having fixed rank.

Lemma 5.4. Let $k, m, n \in \mathbb{N}$ with $k \leq \min \{m, n\}$. Then

$$
p^{(n+m-k) k} \frac{(q)_{n}(q)_{m}}{(q)_{n-k}(q)_{m-k}(q)_{k}}
$$

equals the number of matrices in $\mathbb{F}_{p}^{m \times n}$ of rank $k$.

Making use of this lemma, the number $\theta_{r}$ defined in Lemma 5.3 is easily calculated:

$$
\theta_{r}=p^{\nu r} p^{(\nu+n-r-(n-h-r))(n-h-r)} \frac{(q)_{\nu}(q)_{n-r}}{(q)_{\nu-(n-h-r)}(q)_{h}(q)_{n-h-r}} .
$$

The value $z_{n}$ defined above now takes the form

$$
z_{n}=\sum_{r=n-\nu-h}^{\min \{n-\nu, n-h\}} \theta_{r} \sum_{\substack{\alpha \in \mathcal{R} \\ \exists \gamma_{1}: \operatorname{rk}\left(\gamma_{1}\right)=r \\ g_{1}(\alpha)=\left(\gamma_{1} \mid 0\right)}}\left[\mathrm{M}_{n}(S): \mathrm{M}_{n}(S) \alpha\right]^{-(1+u / n)}
$$

where again $\gamma_{1} \in \mathbb{F}_{p}^{n \times(n-\nu)}$.

Lemma 5.5. Let $n-\nu-h \leq r \leq \min \{n-\nu, n-h\}$. Then

$$
\sum_{\substack{\alpha \in \mathcal{R} \\
\exists \gamma_{1}: \operatorname{rk}\left(\gamma_{1}\right)=r \\
g_{1}(\alpha)=\left(\gamma_{1} \mid 0\right)}}\left[\mathrm{M}_{n}(S): \mathrm{M}_{n}(S) \alpha\right]^{-(1+u / n)}=\left[\begin{array}{c}
n-\nu \\
r
\end{array}\right]_{p} q^{(n+u)(n-r)} \frac{(q)_{u}}{(q)_{n+u-r}},
$$

where again $\gamma_{1} \in \mathbb{F}_{p}^{n \times(n-\nu)}$.

Proof. By Morita's Theorem we can retranslate the sum to a sum over $S$ submodules of $S^{n}$. Thus fix an $r$-dimensional subspace $F \subseteq \mathbb{F}_{p}^{n-\nu}$. Then we will see below that the sum

$$
\sum_{\substack{U \subseteq S^{n} \\ g_{1}(U)=F \oplus 0^{\nu}}}\left[S^{n}: U\right]^{-(n+u)}
$$

does not depend on the particular $F$ chosen. There are in fact $\left[\begin{array}{c}n-\nu \\ r\end{array}\right]_{p}$ choices for $F$, whence the sum to be computed equals

$$
\left[\begin{array}{c}
n-\nu \\
r
\end{array}\right]_{p} \sum_{\substack{U \subseteq S^{n} \\
g_{1}(U)=F \oplus 0^{\nu}}}\left[S^{n}: U\right]^{-(n+u)} .
$$


Since both $S$ and $\mathbb{Z}_{p}$ are discrete valuation rings with residue field $\mathbb{F}_{p}$, and since $g_{1}, g_{2}$ induce isomorphisms $S^{n} / \operatorname{rad}\left(S^{n}\right) \rightarrow \mathbb{F}_{p}^{n}$ and $\mathbb{Z}_{p}^{n} / \operatorname{rad}\left(\mathbb{Z}_{p}^{n}\right) \rightarrow \mathbb{F}_{p}^{n}$ respectively, we get

$\sum_{\substack{U \subseteq S^{n} \\ g_{1}(U)=F \oplus 0^{\nu}}}\left[S^{n}: U\right]^{-(n+u)}=\sum_{\substack{U \subseteq \mathbb{Z}_{p}^{n} \\ g_{2}(U)=F \oplus 0^{\nu}}}\left[\mathbb{Z}_{p}^{n}: U\right]^{-(n+u)}=\sum_{\substack{U \subseteq \mathbb{Z}_{p}^{n} \\ U+p \mathbb{Z}_{p}^{n}=V}}\left[\mathbb{Z}_{p}^{n}: U\right]^{-(n+u)}$

with $p \mathbb{Z}_{p}^{n} \subseteq V \subseteq \mathbb{Z}_{p}^{n}$ such that $V / p \mathbb{Z}_{p}^{n}=F \oplus 0^{\nu}$. By [14, Lemma 7.3] this equals

$$
\begin{aligned}
{\left[\mathbb{Z}_{p}^{n}: V\right]^{-(n+u)} \sum_{\substack{U \subseteq V \\
U+p \mathbb{Z}_{p}^{n}=V}}[V: U]^{-(n+u)} } & =p^{-(n+u)(n-r)} \prod_{j=r}^{n-1}\left(1-q^{n+u-j}\right)^{-1} \\
& =q^{(n+u)(n-r)} \frac{(q)_{u}}{(q)_{n+u-r}} .
\end{aligned}
$$

This proves the lemma.

Now (15) implies

$$
\begin{aligned}
z_{n} & =\sum_{r=n-\nu-h}^{\min \{n-\nu, n-h\}} \theta_{r}\left[\begin{array}{c}
n-\nu \\
r
\end{array}\right]_{p} q^{(n+u)(n-r)} \frac{(q)_{u}}{(q)_{n+u-r}} \\
& =\sum_{r=n-\nu-h}^{\min \{n-\nu, n-h\}} p^{\exp _{r}} \frac{(q)_{\nu}(q)_{n-r}(q)_{n-\nu}(q)_{u}}{(q)_{\nu-(n-h-r)}(q)_{h}(q)_{n-h-r}(q)_{r}(q)_{n-\nu-r}(q)_{n+u-r}}
\end{aligned}
$$

with

$$
\exp _{r}:=-h r+(\nu+h)(n-h)+r(n-\nu-r)-(n+u)(n-r)
$$

as $p$-exponent. Substituting $e:=r-(n-\nu-h)$ yields

$$
z_{n}=\sum_{e=0}^{\min \{\nu, h\}} p^{\exp _{e}^{\prime}} \frac{(q)_{\nu}(q)_{\nu+h-e}(q)_{n-\nu}(q)_{u}}{(q)_{e}(q)_{h}(q)_{\nu-e}(q)_{n-\nu-h+e}(q)_{h-e}(q)_{\nu+h+u-e}}
$$

with

$$
\exp _{e}^{\prime}:=-\left(h^{2}+h u\right)+h(e-\nu)+e \nu+e u-e^{2}-\nu u .
$$

The last step consists in letting $n \rightarrow \infty$, and we get

$$
\begin{aligned}
\lim _{n \rightarrow \infty} z_{n}= & \frac{q^{h(h+\nu+u)+\nu u}(q)_{u}(q)_{\nu}}{(q)_{h}} \\
& \times \sum_{e=0}^{\min \{\nu, h\}} p^{e(\nu+h+u-e)} \frac{(q)_{\nu+h-e}}{(q)_{e}(q)_{\nu-e}(q)_{h-e}(q)_{\nu+h+u-e}} .
\end{aligned}
$$

Now

$$
\lim _{n \rightarrow \infty} x_{n}=\left(\lim _{n \rightarrow \infty} y_{n}\right)\left(\lim _{n \rightarrow \infty} z_{n}\right)
$$


can be derived from Lemma 5.2 and (16). Since by definition $\lim _{n \rightarrow \infty} x_{n}$ equals the limit occuring in Lemma 5.1, the proof of the following main theorem of this section is complete.

Theorem 5.6. Let $G$ be a finite abelian p-group of rank $\nu$, and let $h, u \in \mathbb{N}$. Then

$$
\begin{aligned}
\sum_{\substack{\phi M \cong G \\
\left|\hat{H}^{1}(M)\right|=p^{h}}}\left|\operatorname{Aut}_{R}(M)\right|^{-1}|M|^{-u}= \\
\\
\frac{q^{h(h+\nu+u)+\nu u}(q)_{u}(q)_{\nu}}{(q)_{h}} \kappa(\nu, h, u)|\operatorname{Aut}(G)|^{-1}|G|^{-u},
\end{aligned}
$$

where

$$
\kappa(\nu, h, u):=\sum_{e=0}^{\min \{\nu, h\}} p^{e(\nu+h+u-e)} \frac{(q)_{\nu+h-e}}{(q)_{e}(q)_{\nu-e}(q)_{h-e}(q)_{\nu+h+u-e}} .
$$

We will conclude this section by considering this formula in the special cases $u=0, h=0, \nu=0$ respectively.

Corollary 5.7. Let $G$ be a finite abelian p-group of rank $\nu$, and let $h \in \mathbb{N}$. Then

$$
\sum_{\substack{\phi M \cong G \\\left|\hat{H}^{1}(M)\right|=p^{h}}}\left|\operatorname{Aut}_{R}(M)\right|^{-1}=\frac{q^{h^{2}}}{(q)_{h}^{2}}|\operatorname{Aut}(G)|^{-1} .
$$

Proof. We put $u:=0$ in the preceding theorem, and thus the sum equals

$$
\frac{q^{h(h+\nu)}}{(q)_{h}^{2}}\left(\sum_{e=0}^{\min \{\nu, h\}} p^{e(\nu+h-e)} \frac{(q)_{\nu}(q)_{h}}{(q)_{e}(q)_{\nu-e}(q)_{h-e}}\right)|\operatorname{Aut}(G)|^{-1} .
$$

By Lemma 5.4, the $e$-th term of the expression in brackets equals the number of matrices in $\mathbb{F}_{p}^{\nu \times h}$ of rank e. Hence (17) can be written as

$$
\frac{q^{h(h+\nu)}}{(q)_{h}^{2}}\left|\mathbb{F}_{p}^{\nu \times h}\right||\operatorname{Aut}(G)|^{-1}=\frac{q^{h^{2}}}{(q)_{h}^{2}}|\operatorname{Aut}(G)|^{-1} .
$$

Next we consider the case $h=0$, i.e. the summation extends over cohomologically trivial modules.

Corollary 5.8. Let $G$ be a finite abelian p-group of rank $\nu$, and let $u \in \mathbb{N}$. Then

$$
\sum_{\substack{\phi M \cong G \\ \text { cohom. trivial }}}\left|\operatorname{Aut}_{R}(M)\right|^{-1}|M|^{-u}=q^{\nu u} \frac{(q)_{u}(q)_{\nu}}{(q)_{u+\nu}}|\operatorname{Aut}(G)|^{-1}|G|^{-u} .
$$


Finally let $G=0$.

Corollary 5.9. Let $h, u \in \mathbb{N}$. Then

$$
\begin{aligned}
\sum_{\substack{\phi M=0 \\
\left|\hat{H}^{1}(M)\right|=p^{h}}}\left|\operatorname{Aut}_{R}(M)\right|^{-1}|M|^{-u} & =\sum_{\substack{\phi M=0 \\
|M / I M|=p^{h}}}\left|\operatorname{Aut}_{R}(M)\right|^{-1}|M|^{-u} \\
& =\frac{q^{h(h+u)}(q)_{u}}{(q)_{h}(q)_{h+u}} .
\end{aligned}
$$

\section{References}

[1] C.J. Bushnell, I. Reiner, Zeta functions of arithmetic orders and Solomon's Conjectures. Math. Z. 173 (1980), 135-161.

[2] H. Cohen, H.W. Lenstra, Heuristics on class groups of number fields. Number Theory Noordwijkerhout 1983, LNM 1068, Springer, 1984.

[3] H. Cohen, J. Martinet, Étude heuristique des groupes de classes des corps de nombres. J. reine angew. Math. 404 (1990), 39-76.

[4] S.D. Fisher, M.N. Alexander, Matrices over a finite field. Am. Math. Monthly 73 (1966), 639-641.

[5] C. Greither, Galois-Cohen-Lenstra heuristics. Acta Math. et Inf. Univ. Ostraviensis 8 (2000), 33-43.

[6] P. Hall, A partition formula connected with Abelian groups. Comment. Math. Helv. 11 (1938/39), 126-129.

[7] G.H. Hardy, E.M. Wright, An Introduction to the Theory of Numbers. Oxford University Press, 1979

[8] B. Huppert, Endliche Gruppen I. Springer, 1967.

[9] N. Jacobson, Basic Algebra II. Freeman, 1980.

[10] I. Reiner, Zeta functions of integral representations. Comm. Algebra 8 (1980), 911-925.

[11] G.-C. RотA, On the foundations of combinatorial theory I. Theory of Möbius functions. Z. Wahrscheinlichkeitstheorie 2 (1964), 340-368.

[12] J.-P. Serre, Local Fields. Springer, 1995.

[13] L. Solomon, Zeta functions and integral representation theory. Adv. Math. 26 (1977), 306-326.

[14] C. Wittmann, Zeta functions of integral representations of cyclic p-groups. J. Algebra 274 (2004), 271-308.

[15] C. Wittmann, p-class groups of certain extensions of degree p. Math. Comp. 74 (2005), 937-947.

Christan WitTmanN

Universität der Bundeswehr München

Fakultät für Informatik

Institut für Theoretische Informatik und Mathematik

85577 Neubiberg, Germany

E-mail : wittmann@informatik.unibw-muenchen.de 